\title{
Fazit und Ausblick
}

Der Projektkurs „Selberdenken!“ zeigt Möglichkeiten auf, wie mit Hilfe einer naturphilosophischen Betrachtungsweise naturwissenschaftlich-technischer Themen ein tiefergehendes, authentisches Bild der Naturwissenschaften gewonnen werden kann. Vor allem ein explizites Thematisieren von Aspekten zur Natur der Naturwissenschaften, ein wissenschaftstheoretisches Hinterfragen der Struktur, Dynamik und Bedingungen der Naturwissenschaften sowie deren kultureller und historischer Bedingtheit wirkt Fehlvorstellungen entgegen. Von besonderer Bedeutung ist dabei, dass die Schülerinnen und Schüler durch forschend-entdeckendes Lernen und exploratives Experimentieren den Forschungsprozess selbst erfahren und Grundlagen der wissenschaftlichen Denk- und Arbeitsweise erkunden können. Auf diese Weise wird die Basis für eine kritisch-neugierige Sichtweise auf die Welt geschaffen, die nicht nur beim Erlernen von Fachinhalten von Bedeutung ist, sondern die Basis für ein mündiges Erfassen und Gestalten unserer technisierten Welt darstellt.

„Selberdenken!““ ist ein Pilotprojekt, welches als Vorbild für weitere Tandems aus Schule und Hochschule dienen und sie ermutigen soll, mit einer naturphilosophischen Perspektive auf die Naturwissenschaften mündig agierende Jugendliche heranzubilden. 